\section{Comment on "An Apparent General Solution for the Kinetic Models of the Bacteriorhodopsin Photocycles"}

\author{
Richard H. Lozier* and John F. Nagle \\ Department of Physics, Carnegie-Mellon University, \\ Pittsburgh, Pennsylvania 15213
}

\section{Received: October 18, 2005; In Final Form: June 2, 2006}

Richard Hendler has presented a detailed comparison of reversible homogeneous models (RHM) and unidirectional parallel models (UPM) for the bacteriorhodopsin photocycle ${ }^{1}$ using data from his own laboratory ${ }^{2}$ and the data of Xie et al. ${ }^{3}$ He makes an apparently valid criticism of the analysis of the latter data as fitted to an RHM model ( $\mathrm{K} \leftrightarrow \mathrm{L} \leftrightarrow \mathrm{M}_{1} \leftrightarrow \mathrm{M}_{2} \leftrightarrow$ $\mathrm{N} \leftrightarrow \mathrm{O} \rightarrow$ bR) by van Stokkum and Lozier ${ }^{4}$ that we will discuss in the next paragraph. Unfortunately, he fails to appreciate that the polarization of measuring and actinic beams must be properly set to avoid kinetics of physical motions of the excited and unexcited chromophores admixing with the absorption changes due to the photochemical cycle, as has been shown by many authors, ${ }^{5-12}$ and thus his own analysis is seriously flawed. This will be discussed in turn below.

First, we will discuss Hendler's criticism of van Stokkum and Lozier's analysis, ${ }^{4}$ add our own additional criticism to his, and provide some rationalization mitigating both. Hendler states in the abstract of his paper that "... the tested RHM does not really work for the data of van Stokkum and Lozier ...". By this he apparently means that his "Requirement 3 . The eigenvalues of the J-matrix must be equal to the kinetic macro constants obtained by fitting exponentials to the experimental data." (page 16525 of Hendler's article) is not met. Our additional criticism of van Stokkum and Lozier's analysis ${ }^{4}$ is that, although on the scale of the data the model fits the data rather well with a single simple model that accommodates data taken over a wide range of conditions (47 times spaced logarithmically from $1 \mu \mathrm{s}$ to $300 \mathrm{~ms}$, three polarization conditions (parallel, perpendicular, and magic angle), 15 wavelengths from 380 to $700 \mathrm{~nm}$, seven temperatures from 5 to $35^{\circ} \mathrm{C}$, three $\mathrm{pH}$ values $(5,7$, and 9$)$, and in $\mathrm{D}_{2} \mathrm{O}$ medium at $\mathrm{pD}=7$, see Figures 1 and 2 of ref 4 ), some residuals (Figure 3 of ref 4 ) are as large as $3 \mathrm{~mA}$ (for comparison, the maximal signals of the data exceed $50 \mathrm{~mA}$, Figure 1 of ref 4). The fitting of exponentials to subsets of the data at all wavelengths but single temperature, $\mathrm{pH}$ value, and polarization has many fewer constraints (fewer data points per fitted parameter) than the fitting of the RHM model of ref 4, and the maximal residuals for a six exponential fit are only $0.5 \mathrm{~mA}$ (Figure 3e of ref 3). The rationale mitigating this is that the analysis of ref 4 requires only one parameter per 76.4 data points, whereas the seven exponential fits of ref 3 and of Hendler's current paper requires one parameter per 6.29 data points. At pH 7 and $10^{\circ} \mathrm{C}$, Hendler fits the data to nine exponentials (page 16520 of ref 1), resulting in only 5.22 data points per parameter. Hendler has much greater confidence in our data than we do! A fact of life is that data can be more precisely fit by increasing the number of parameters, but the physical meaning of the parameters become more

* Corresponding author e-mail: lozier@pentabox.ru. obscure with increasing number of parameters, as will be discussed below for Hendler's paper.

Now to the issue of Hendler's incorrect use of our data taken using polarizers. The theoretical basis for choice of polarization conditions was established long ago. ${ }^{13}$ Briefly explained, three polarization conditions are commonly used to investigate kinetics of both physical motions and chemical transitions: parallel, perpendicular, and magic angle (see Supporting Information, Figure 1 and its discussion). When the polarizations of measuring and actinic beams are parallel, one measures signals from the chemical transitions and from rotations of excited chromophores out of and nonexcited chromophores into the polarization of the measuring beam. When the polarizations of measuring and actinic beams are perpendicular, one measures signals from the chemical transitions and from rotations of excited chromophores into and nonexcited chromophores out of the polarization of the measuring beam. Under magic angle polarization conditions (for small absorption changes discussed here $^{14}$ ) rotations of excited and nonexcited chromophores into and out of the polarization of the measuring beam cancel each other, so the net signal is due only to the photochemical absorption changes. The absorption changes due to physical motions only can be obtained from the polarization anisotropy

$$
\left(\mathrm{A}_{\text {parallel }}-\mathrm{A}_{\text {perpendicular }}\right) /\left(\mathrm{A}_{\text {parallel }}+2 \mathrm{~A}_{\text {perpendicular }}\right)
$$

although the interpretation involves nontrivial considerations ${ }^{11}$ not appreciated by Dr Hendler that we discuss in the Supporting Information (see Supporting Information Figure 2 and its discussion). In two limiting cases, (i) when the chromophores randomize in space rapidly with respect to the fastest time frame of the absorption measurement or (ii) when the chromophores are fixed in space for times longer than the slowest time frame of the absorption measurement, fixed polarizations are not important. These conditions hold for the vast majority of optical spectroscopic measurements with true solutions (case i) or for colored glasses (case ii). But for the time frame under consideration here (microsecond to subsecond times), physical motions of bacteriorhodopsin in purple membrane suspensions do occur, ${ }^{5-12}$ so appropriate polarization conditions are essential. The data files provided by Lozier to Hendler contained the three polarization conditions, and Hendler chose to use the parallel data which are admixed with motional information (see Supporting Information Figure 1 and its discussion). Hendler states in the first paragraph of the "Methods" section of his paper ( $p$ 16516) "Data acquired under parallel polarization conditions were used, to be compatible with most published kinetic studies on the BR photocycle". Although he is correct that some authors have overlooked this detail (e.g., ref 15), many investigators have used the appropriate polarization conditions. ${ }^{5-14}$ Hendler apparently believes, incorrectly, that data taken without defined polarization should be most similar to the parallel case. In fact, if neither measuring nor actinic beam were polarized, the result would be most similar (though still not identical) to the magic angle case. However, optical spectroscopy is subject to stray polarization (e.g., from asymmetry of the lamp filament and envelope and from dispersive elements of monochromators), and the extent and direction of polarization is wavelength dependent, ${ }^{7}$ a fact too often overlooked ${ }^{1,2,15}$.

It is easily seen in the data set of Xie et al. ${ }^{3}$ that the normalized parallel and magic angle data differ over the whole 
time range and with a maximum difference amplitude of about $2 \mathrm{~mA}$ (Figure 1 of the Supporting Information). Thus, Hendler's paper fits a motional artifact with amplitudes comparable to the largest residuals of the RHM fit of ref 4 ! We encouraged Dr Hendler to perform his analysis using the appropriate (magic angle) data. In a personal communication he wrote "the time constants for the transition events are different between the $\mathrm{A}_{\text {parallel }}$ and $\mathrm{A}_{\mathrm{MA}}$ data, and ... although we can obtain a multicycle solution for the $\mathrm{A}_{\text {parallel }}$ data at $\mathrm{pH} 7$ and $20^{\circ} \mathrm{C}$, we could not do the same with the $\mathrm{A}_{\mathrm{MA}}$ data". This confirms that it matters which data are used in the analysis. We also conclude that Hendler's multicycle UPM model is incorrect for the bacteriorhodopsin photocycle.

Supporting Information Available: Additional discussion and figures. This material is available free of charge via the Internet at http://pubs.acs.org.

\section{References and Notes}

(1) Hendler, R. W. J. Phys. Chem. B 2005, 109, 16515-16528.
(2) Hendler, R. W.; Shrager, R. I.; Bose, S. J. Phys. Chem. B 2001, $105,3319-3328$ 635.

(3) Xie, A. H.; Nagle, J. F.; Lozier, R. H. Biopys. J. 1987, 51, 627-

(4) van Stokkum, I. H. M.; Lozier, R. H. J. Phys. Chem. B 2002, 106, $3477-3485$

(5) Slifkin, M. A.; Caplan, S. R. Nature 1975, 253, 56-58.

(6) Lozier, R. H.; Niederberger, W. Fed. Proc. 1977, 36, 1805-1809.

(7) Lozier, R. H. Methods Enzymol. 1982, 88, 133-162.

(8) Nagle, J. F.; Parodi, L. A.; Lozier, R. H. Biophys. J. 1982, 38, $161-174$.

(9) Ahl, P. L.; Cone, R. A. Biophys. J. 1984, 45, 1039-1049.

(10) Zimanyi, L.; Keszthelyi, L.; Lanyi, J. K. Biochemistry 1989, 28, $5165-5172$.

(11) Wan, C.; Qian, J.; Johnson, C. K. Biophys. J. 1993, 65, 927-938.

(12) Borucki, B.; Otto, H.; Heyn, M. P. J. Phys. Chem. B 1999, 103, $6371-6383$

(13) Albrecht, A. C. J. Mol. Spectrosc. 1961, 6, 84-108. 968.

(15) Chizhov, I.; Chernavskii, D. S.; Engelhard, M.; Mueller, K.-H.; Zubov, B. V.; Hess, B. Biophys. J. 1996, 71, 2329-2345. 